PROCEEDINGS OF THE

AMERICAN MATHEMATICAL SOCIETY

Volume 126, Number 4, April 1998, Pages 1071-1080

S 0002-9939(98)04253-1

\title{
THE STABILITY RADIUS OF A QUASI-FREDHOLM OPERATOR
}

\author{
PAK WAI POON
}

(Communicated by Palle E. T. Jorgensen)

\begin{abstract}
We extend the technique used by Kordula and Müller to show that the stability radius of a quasi-Fredholm operator $T$ is the limit of $\gamma\left(T^{n}\right)^{1 / n}$ as $n \rightarrow \infty$. If 0 is an isolated point of the Apostol spectrum $\sigma_{\gamma}(T)$, then the above limit is non-zero if and only if $T$ is quasi-Fredholm.
\end{abstract}

Let $L(X)$ be the set of all bounded linear operators on a complex Banach space $X$. For any $T \in L(X)$, we denote the null space and range of $T$ by $N(T)$ and $R(T)$ respectively. The Apostol spectrum of $T$ is defined to be the set

$$
\sigma_{\gamma}(T)=\left\{\mu \in \mathbb{C}: \lim _{\lambda \rightarrow \mu} \gamma(T-\lambda I)=0\right\},
$$

where $\gamma(T)$ is the reduced minimum modulus of $T$, that is,

$$
\gamma(T)= \begin{cases}\inf \{\|T x\|: x \in X, d(x, N(T))=1\} & \text { if } T \neq 0 \\ \infty & \text { if } T=0\end{cases}
$$

The Apostol spectrum was first defined in this form by Apostol in [1] for operators on a Hilbert space. Its complement in $\mathbb{C}$ is usually called the semi-regular region of $T$ and is denoted by $\rho_{\gamma}(T)$. $T$ is semi-regular (or s-regular) if $0 \in \rho_{\gamma}(T)$. Properties of the Apostol spectrum for operators on a Banach space can be found in [10,11]. The stability radius of $T$ is defined as the distance

$$
\delta(T)=d\left(0, \sigma_{\gamma}(T) \backslash\{0\}\right) .
$$

It is the radius of the largest punctured open disc centred at 0 in which $T-\lambda I$ is semi-regular. When $T$ is semi-regular, it is shown in [5] that

$$
\delta(T)=\lim _{n \rightarrow \infty} \gamma\left(T^{n}\right)^{1 / n} .
$$

In the special case when $T$ is bounded below or surjective, $\delta(T)$ is also the distance from 0 to the approximate point spectrum and to the surjectivity spectrum respectively. When 0 is an isolated point of $\sigma_{\gamma}(T)$, the formula (3) still applies to certain classes of operators. It includes the cases when $T$ is Fredholm [3], semi-Fredholm [14], essentially s-regular [5], or chain finite ( $T$ has finite ascent and descent) [2].

Received by the editors June 21, 1996 and, in revised form, September 23, 1996.

1991 Mathematics Subject Classification. Primary 47A55, 47A10, 47A53.

Key words and phrases. Stability radius, Apostol spectrum, semi-regular, quasi-Fredholm operators, ascent, descent.

The results in this paper form a part of the author's research for the degree of $\mathrm{Ph}$. $\mathrm{D}$. at the University of Melbourne, 1996, under the supervision of J. J. Koliha.

(C)1998 American Mathematical Society 
An operator $T$ is regular (or of Saphar type) if it is both relatively regular and semi-regular. The stability problem concerning regular operators is studied in [13].

Definition. An operator $T \in L(X)$ has topological uniform descent $d$ (where $d$ is a nonnegative integer) if $N\left(T^{n}\right)+R(T)=N\left(T^{d}\right)+R(T)$ is a closed subspace for all $n \geq d$ (Grabiner [4]). An operator $T$ is quasi-Fredholm if $T$ has topological uniform descent $d$ for some integer $d$ and $R\left(T^{n}\right)$ is closed for all $n \geq d$.

Quasi-Fredholm operators were first defined on a Hilbert space by Labrousse [6] and on a Banach space by Mbekhta and Müller [9]. The definition used here is different from but equivalent to the one given in [9]. The class of quasi-Fredholm operators is well researched for the Hilbert space case [6, 7]. In [6], it was shown that an operator on a Hilbert space is quasi-Fredholm if and only if it has a Kato decomposition. A characterization of quasi-Fredholm operators in a Banach space is examined in [12]. Two pertinent properties of quasi-Fredholm operators are proved in Theorem 7 and Corollary 15 below.

In the present paper, the stability radius problem of a quasi-Fredholm operator is examined. It was shown in [1, Prop. 3.3] that the stability radius formula (3) holds for a Hilbert space operator $T$ if only and if $T$ has a Kato decomposition described in [6], which is of course equivalent to $T$ being quasi-Fredholm. The main aim of this paper is to extend the result to Banach space operators. It turns out that the technique used by Kordula and Müller in [5] can be extended to solve the stability radius problem for quasi-Fredholm operators. The main result of this paper is the following theorem, which is a consequence of Theorem 10 and Corollary 15.

Theorem. If $T$ is a quasi-Fredholm operator, then the stability radius of $T$ is equal to $\lim _{n \rightarrow \infty} \gamma\left(T^{n}\right)^{1 / n}$. Moreover, if 0 is an isolated point of the Apostol spectrum of an arbitrary operator $T$, then $\lim _{n \rightarrow \infty} \gamma\left(T^{n}\right)^{1 / n}$ always exists. This limit is nonzero if and only if $T$ is quasi-Fredholm.

If $M, N$ are closed $T$-invariant subspaces with $N \subseteq M$, then we denote the map induced by $T$ on the quotient $M / N$ by $T_{M / N}$. More precisely, $T_{M / N}$ is the map $x+N \mapsto T x+N$. We also denote the restriction of $T$ to $M$ by $T_{M}$. The ascent and descent of $T$ will be denoted by $\operatorname{asc}(T)$ and $\operatorname{des}(T)$ respectively. The hyperkernel $\bigcup_{n=1}^{\infty} N\left(T^{n}\right)$ and hyperrange $\bigcap_{n=1}^{\infty} R\left(T^{n}\right)$ of $T$ are denoted by $N\left(T^{\infty}\right)$ and $R\left(T^{\infty}\right)$ respectively. We first prove some properties of the reduced minimum modulus.

Lemma 1. Let $M$ be a closed T-invariant subspace.

(i) If $T_{X / M}$ is injective, then $\gamma\left(T_{M}\right) \geq \gamma(T)$.

(ii) If $T_{M}$ has dense range, then $\gamma\left(T_{X / M}\right) \geq \gamma(T)$.

Proof. (i) The hypothesis shows that $T^{-1} M \subseteq M$. In particular, $N(T) \subseteq M$. Hence $N\left(T_{M}\right)=N(T) \cap M=N(T)$. It is clear from the definition of the minimum modulus that $\gamma\left(T_{M}\right) \geq \gamma(T)$.

(ii) If $\gamma\left(T_{X / M}\right)=\infty$, the result is trivial. If $\gamma>\gamma\left(T_{X / M}\right)$, we can find $x \in X$ such that $d\left(x+M, N\left(T_{X / M}\right)\right)=1$ and $\left\|T_{X / M}(x+M)\right\|<\gamma$. So

$$
d\left(x, T^{-1} M\right)=1 \quad \text { and } \quad d(T x, M)<\gamma .
$$

Since $T M$ is dense in $M$, we can find $m \in M$ such that $\|T(x+m)\|<\gamma$. Note that $N(T) \subseteq T^{-1} M$. As $T M \subseteq M$, we also have $M \subseteq T^{-1} M$. Hence

$$
\gamma(T) \leq \frac{\|T(x+m)\|}{d(x+m, N(T))} \leq \frac{\|T(x+m)\|}{d\left(x+m, T^{-1} M\right)}=\frac{\|T(x+m)\|}{d\left(x, T^{-1} M\right)}<\gamma .
$$


Considering all $\gamma>\gamma\left(T_{X / M}\right)$, we conclude that $\gamma\left(T_{X / M}\right) \geq \gamma(T)$.

Lemma 2. Suppose $\operatorname{asc}(T)=$ d, i.e. $N\left(T^{d}\right)=N\left(T^{n}\right)$ for all $n \geq d$. Let $\widehat{T}$ be the map induced by $T$ on the quotient $X / N\left(T^{d}\right)$; then

$$
\begin{aligned}
& \liminf _{n \rightarrow \infty} \gamma\left(T^{n}\right)^{1 / n}=\liminf _{n \rightarrow \infty} \gamma\left(\widehat{T}^{n}\right)^{1 / n}, \\
& \limsup _{n \rightarrow \infty} \gamma\left(T^{n}\right)^{1 / n}=\limsup _{n \rightarrow \infty} \gamma\left(\widehat{T}^{n}\right)^{1 / n} .
\end{aligned}
$$

Moreover, $\limsup _{n \rightarrow \infty} \gamma\left(T^{n}\right)^{1 / n}>0 \Longleftrightarrow \widehat{T}$ is bounded below $\Longleftrightarrow \lim _{n \rightarrow \infty} \gamma\left(T^{n}\right)^{1 / n}>0$.

Proof. Let us first assume that $T^{d} \neq 0$. Since $\operatorname{asc}(T)=d$, we have $T^{-1} N\left(T^{d}\right)=$ $N\left(T^{d}\right)$ and $\widehat{T}$ is injective. Taking any $x \in X$, we have

$$
\left\|T^{n} x\right\| \geq d\left(T^{n} x, N\left(T^{d}\right)\right) \geq\left\|T^{d}\right\|^{-1}\left\|T^{n+d} x\right\| .
$$

The first inequality is obvious. The second follows from the relation

$$
\left\|T^{n+d} x\right\|=\left\|T^{d}\left(T^{n} x+z\right)\right\| \leq\left\|T^{d}\right\|\left\|T^{n} x+z\right\|,
$$

true for all $z \in N\left(T^{d}\right)$. Write $x+N\left(T^{d}\right)$ as $\widehat{x}$; then for $n \geq d$, we have

$$
\left\|\widehat{T}^{n} \widehat{x}\right\|=d\left(T^{n} x, N\left(T^{d}\right)\right), \quad\|\widehat{x}\|=d\left(x, N\left(T^{d}\right)\right)=d\left(x, N\left(T^{n}\right)\right)=d\left(x, N\left(T^{n+d}\right)\right) .
$$

We deduce from (5) that $\gamma\left(T^{n}\right) \geq \gamma\left(\widehat{T}^{n}\right) \geq\left\|T^{d}\right\|^{-1} \gamma\left(T^{n+d}\right)$ by taking infima over all $x$ with $\|\widehat{x}\|=1$; (4) then follows by taking limits. When $T^{d}=0, \widehat{T}=0$ and all limits in (4) are infinite. To establish the last statement, we proceed as follows:

(a) If $\lim \sup _{n \rightarrow \infty} \gamma\left(T^{n}\right)^{1 / n}>0$, then it follows from (4) that $\gamma\left(\widehat{T}^{k}\right)>0$ for some $k$. Therefore $R\left(\widehat{T}^{k}\right)$ is a closed subspace. Since $\widehat{T}$ is injective, so is $\widehat{T}^{k}$. Thus $\widehat{T}^{k}$ is injective and has closed range. So $\widehat{T}^{k}$ is bounded below. Hence the Banach space adjoints of $\widehat{T}^{k}$ and $\widehat{T}$ are surjective. So $\widehat{T}$ is bounded below.

(b) If $\widehat{T}$ is bounded below, then the $\operatorname{limit}_{\lim } \rightarrow \infty \gamma\left(\widehat{T}^{n}\right)^{1 / n}$ exists and is positive [8]. By (4), the same is true for $\lim _{n \rightarrow \infty} \gamma\left(T^{n}\right)^{1 / n}$.

(c) If $\lim _{n \rightarrow \infty} \gamma\left(T^{n}\right)^{1 / n}>0$, then obviously $\lim \sup _{n \rightarrow \infty} \gamma\left(T^{n}\right)^{1 / n}>0$.

By applying the lemma to $T^{*}$ instead of $T$ and using Banach space duality, one can readily verify the following lemma.

Lemma 3. Suppose $\overline{R\left(T^{n}\right)}=\overline{R\left(T^{d}\right)}$ for all $n \geq d$. Let $\widetilde{T}$ be the restriction of $T$ to $\overline{R\left(T^{d}\right)}$; then

$$
\begin{aligned}
& \liminf _{n \rightarrow \infty} \gamma\left(T^{n}\right)^{1 / n}=\liminf _{n \rightarrow \infty} \gamma\left(\widetilde{T}^{n}\right)^{1 / n}, \\
& \limsup _{n \rightarrow \infty} \gamma\left(T^{n}\right)^{1 / n}=\limsup _{n \rightarrow \infty} \gamma\left(\widetilde{T}^{n}\right)^{1 / n} .
\end{aligned}
$$

Moreover, $\limsup _{n \rightarrow \infty} \gamma\left(T^{n}\right)^{1 / n}>0 \Longleftrightarrow \widetilde{T}$ is surjective $\Longleftrightarrow \lim _{n \rightarrow \infty} \gamma\left(T^{n}\right)^{1 / n}>0$.

The following lemma is a refinement of Kordula and Müller [5, Lemma 2]. The technique used in the proof is adapted from that paper.

Lemma 4. Let $N \subseteq M$ be closed $T$-invariant subspaces of $X$ such that $T_{X / M}$ is bounded below, $T_{N}$ is surjective and $T^{d} M \subseteq N$. Then:

(i) $N=R\left(T^{n}\right) \cap M=T^{n} M$ for each $n \geq d$.

(ii) $M=N\left(T^{n}\right)+N=T^{-n} N$ for each $n \geq d$. 
(iii) $\operatorname{des}\left(T_{M}\right) \leq d, R\left(T_{M}^{d}\right)=N$ and

$$
\lim _{n \rightarrow \infty} \gamma\left(T_{M}^{n}\right)^{1 / n}=\lim _{n \rightarrow \infty} \gamma\left(T_{N}^{n}\right)^{1 / n} .
$$

(iv) $\operatorname{asc}\left(T_{X / N}\right) \leq d, N\left(T_{X / N}^{d}\right)=M / N$ and

$$
\lim _{n \rightarrow \infty} \gamma\left(T_{X / N}^{n}\right)^{1 / n}=\lim _{n \rightarrow \infty} \gamma\left(T_{X / M}^{n}\right)^{1 / n} .
$$

(v) $\lim _{n \rightarrow \infty} \gamma\left(T^{n}\right)^{1 / n}=\min \left\{\lim _{n \rightarrow \infty} \gamma\left(T_{X / M}^{n}\right)^{1 / n}, \lim _{n \rightarrow \infty} \gamma\left(T_{N}^{n}\right)^{1 / n}\right\}$.

Proof. Consider any integer $n \geq d$.

(i) Since $T_{X / M}$ is injective, we have $T^{-1} M=M$. So $T^{n} M=T^{n} T^{-n} M=$ $M \cap R\left(T^{n}\right)$. From the fact that $T_{N}$ is surjective, $N \subseteq M$ and $T^{d} M \subseteq N$, we have the inclusions $N \subseteq T^{n} N \subseteq T^{n} M \subseteq N$. This establishes (i).

(ii) Since $T_{N}$ is surjective, we have $T^{-n} N=T^{-n} T^{n} N=N\left(T^{n}\right)+N$. From the fact that $T^{n} M \subseteq N, N \subseteq M$ and $T_{X / M}$ is injective, we have the inclusions $M \subseteq T^{-n} N \subseteq T^{-n} M \subseteq M$. This shows (ii).

(iii) It follows from (i) that $\operatorname{des}\left(T_{M}\right) \leq d, R\left(T_{M}^{d}\right)=N$. Using the notation in Lemma 3, we have $\left(T_{M}\right)^{\sim}=T_{N}$, a surjective operator by hypothesis. The rest of (iii) follows from an application of Lemma 3 to the operator $T_{M}$.

(iv) It follows from (ii) that $N\left(T_{X / N}^{n}\right)=T^{-n} N / N=M / N$ for all $n \geq d$. So $\operatorname{asc}\left(T_{X / N}\right) \leq d$ and $N\left(T_{X / N}^{d}\right)=M / N$. Using the notation in Lemma 2, we can identify $\left(T_{X / N}\right)^{\wedge}$ with $T_{X / M}$, which is bounded below. The rest of (iv) follows from an application of Lemma 2 to the operator $T_{X / N}$.

(v) From (iii), (iv) and Lemma 1,

$$
\limsup _{n \rightarrow \infty} \gamma\left(T^{n}\right)^{1 / n} \leq \min \left\{\lim _{n \rightarrow \infty} \gamma\left(T_{X / M}^{n}\right)^{1 / n}, \lim _{n \rightarrow \infty} \gamma\left(T_{N}^{n}\right)^{1 / n}\right\} .
$$

To prove the reverse inequality, we adopt the approach of [5, Lemma 2]. We assume that $M \neq X$ and $N \neq 0$; otherwise, (v) follows from (iii) and (iv) directly. This means that $T_{N}, T_{X / M}$ are non-zero operators which are either surjective or bounded below. Hence, both $\gamma\left(T_{N}^{n}\right), \gamma\left(T_{X / M}^{n}\right)$ are finite and positive for all $n$. Since (v) holds if and only if it holds for some non-zero multiple of $T$, we can also assume without loss of generality that $\|T\|=1$. For each $i \geq d$, let $\gamma_{i}^{-1}$ be the maximum of $\gamma\left(T_{N}^{i}\right)^{-1}$ and $\gamma\left(T_{X / M}^{i}\right)^{-1}$. We also let $t>1$ and $n \geq d$, and we let $x$ be an arbitrary unit vector in $R\left(T^{n}\right)$. For each $i=d, \ldots, n$, it is possible to pick $x_{i} \in T^{-i}[x+M]$ such that $\left\|x_{i}\right\| \leq t d\left(x_{i}, M\right)$. Since $d\left(x_{i}, M\right) \leq \gamma\left(T_{X / M}^{i}\right)^{-1} d(x, M)$,

$$
\left\|x_{i}\right\| \leq t \gamma\left(T_{X / M}^{i}\right)^{-1}\|x\| \leq t \gamma_{i}^{-1} .
$$

Let $m_{i}=T x_{i+1}-x_{i}$ for $i=d, \ldots, n-1$; then

$$
\left\|m_{i}\right\| \leq\left\|T x_{i+1}\right\|+\left\|x_{i}\right\| \leq\left\|x_{i+1}\right\|+\left\|x_{i}\right\| \leq t\left(\gamma_{i+1}^{-1}+\gamma_{i}^{-1}\right) .
$$

and $\sum_{i=d}^{n-1} T^{i} m_{i}+T^{d} x_{d}-x=T^{n} x_{n}-x$. It is clear from the definition of $x_{i}$ that $T^{i} m_{i} \in M$. So $m_{i} \in T^{-i} M \subseteq M$. If $i \geq d$, then $T^{d} m_{i} \in R\left(T^{d}\right) \cap M=N$ by (i). As $T_{N}$ is surjective, there exists $u_{i} \in N$ such that $T^{d} m_{i}=T^{n-i+d} u_{i}$ and $\left\|u_{i}\right\| \leq t d\left(u_{i}, N\left(T^{n-i+d}\right)\right)$. Therefore,

$$
\left\|u_{i}\right\| \leq t \gamma\left(T_{N}^{n-i+d}\right)^{-1}\left\|T^{d} m_{i}\right\| \leq t \gamma_{n-i+d}^{-1}\left\|m_{i}\right\| \leq t^{2} \gamma_{n-i+d}^{-1}\left(\gamma_{i+1}^{-1}+\gamma_{i}^{-1}\right) .
$$


It is easy to verify that $T^{i} m_{i}=T^{n} u_{i}$. Let $m=T^{d} x_{d}-x$. Since $x \in R\left(T^{n}\right)$, we have $m \in R\left(T^{d}\right) \cap M=N$. We can pick $u \in N$ with $m=T^{n} u,\|u\| \leq t d\left(u, N\left(T_{N}^{n}\right)\right)$,

$$
\|u\| \leq t \gamma\left(T_{N}^{n}\right)^{-1}\|m\| \leq t \gamma_{n}^{-1}\|m\| \leq t^{2} \gamma_{n}^{-1}\left(\gamma_{d}^{-1}+1\right) .
$$

Let $z=x_{n}-\sum_{i=d}^{n-1} u_{i}-u$. We now have

$$
\begin{gathered}
T^{n} z=T^{n} x_{n}-\sum_{i=d}^{n-1} T^{i} m_{i}-\left(T^{d} x_{d}-x\right)=x \\
d\left(z, N\left(T^{n}\right)\right) \leq\|z\| \leq C\left[\gamma_{n}^{-1}+\sum_{i=d}^{n-1} \gamma_{n-i+d}^{-1}\left(\gamma_{i+1}^{-1}+\gamma_{i}^{-1}\right)+\gamma_{n}^{-1}\left(\gamma_{d}^{-1}+1\right)\right]
\end{gathered}
$$

where $C$ is the constant $\max \left\{t, t^{2}\right\}$, which is independent of $n$ and $x$. Since $x$ is an arbitrary unit vector in $R\left(T^{n}\right)$, we have

$$
\gamma\left(T^{n}\right)^{-1} \leq C\left[\gamma_{n}^{-1}+\sum_{i=d}^{n-1} \gamma_{n-i+d}^{-1}\left(\gamma_{i+1}^{-1}+\gamma_{i}^{-1}\right)+\gamma_{n}^{-1}\left(\gamma_{d}^{-1}+1\right)\right] .
$$

Suppose $0<\gamma<\min \left\{\lim _{n \rightarrow \infty} \gamma\left(T_{X / M}^{n}\right)^{1 / n}, \lim _{n \rightarrow \infty} \gamma\left(T_{N}^{n}\right)^{1 / n}\right\}$; then for large enough $i$, say $i \geq n_{0}$, we have $\gamma^{-i} \geq \gamma_{i}^{-1}$. Let $K=1+\max _{d \leq i \leq n_{0}} \gamma^{i} \gamma_{i}^{-1}$; then $\gamma_{i}^{-1} \leq K \gamma^{-i}$ for all $i$. It is a routine calculation that

$$
\begin{aligned}
\gamma\left(T^{n}\right)^{-1} & \leq C K^{2}\left[\gamma^{-n}+\sum_{i=d}^{n-1}\left(\gamma^{-n-d-1}+\gamma^{-n-d}\right)+\gamma^{-n-d}+\gamma^{-n}\right] \\
& \leq \gamma^{-n} C K^{2}(3+2 n-2 d) \max \left\{1, \gamma^{-d}, \gamma^{-d-1}\right\}
\end{aligned}
$$

Taking limits, we have $\liminf _{n \rightarrow \infty} \gamma\left(T^{n}\right)^{1 / n} \geq \gamma$. By considering all possible $\gamma$, we have

$$
\liminf _{n \rightarrow \infty} \gamma\left(T^{n}\right)^{1 / n} \geq \min \left\{\lim _{n \rightarrow \infty} \gamma\left(T_{X / M}^{n}\right)^{1 / n}, \lim _{n \rightarrow \infty} \gamma\left(T_{N}^{n}\right)^{1 / n}\right\} .
$$

Using the lemma, the stability radius formula can be proved via the Apostol representation for quasi-Fredholm operator [12]. For the sake of completeness, we give an independent proof of the result.

Lemma 5 ([5, Lemma 1]). $T$ is s-regular if and only if there exists a closed subspace $M$ with $T M=M$ and $T_{X / M}$ bounded below. We may choose $M$ to be $R\left(T^{\infty}\right)$.

Lemma 6. Let $T$ be quasi-Fredholm; then $\delta(T)>0$. If $\Omega$ is the component of $\rho_{\gamma}(T)$ containing $\{\lambda: 0<|\lambda|<\delta(T)\}$ and $d$ is the uniform descent of $T$, then

$$
R\left[(T-\lambda I)^{\infty}\right]=R\left(T^{\infty}\right)+N\left(T^{\infty}\right)=R\left(T^{\infty}\right)+N\left(T^{d}\right)
$$

for all $\lambda \in \Omega$.

Proof. See [4, Theorem 4.7], and note that $T-\lambda I$ has closed range and uniform descent for $n \geq 0$ if and only if $\lambda \in \rho_{\gamma}(T)$ [10, Corollaire 4.2 (iii)].

Theorem 7. $T$ is a quasi-Fredholm operator if and only if there exist closed $T$ invariant subspaces $M, N$ with $N \subseteq M$ such that $T_{X / M}$ is bounded below, $T_{N}$ is surjective and $T^{d} M \subseteq N$ for some nonnegative integer $d$. We may take $N=R\left(T^{\infty}\right)$ and $M=N\left(T^{d}\right)+R\left(T^{\infty}\right)$, where $d$ is the uniform descent of $T$. 
Proof. Suppose there are subspaces $M, N$ with the required properties; then the requirements for Lemma 4 are satisfied. In particular, $N=R\left(T^{n}\right) \cap M$ and $M=$ $N\left(T^{n}\right)+N$ for each $n \geq d$. As $T_{X / M}$ is bounded below, $R\left(T_{X / M}\right)$ is closed. So is $R(T)+M$. Since

$$
R(T)+M=R(T)+N\left(T^{n}\right)+N=R(T)+N\left(T^{n}\right) \text { for all } n \geq d,
$$

$T$ has topological uniform descent for $n \geq d$.

It remains to show that $R\left(T^{n}\right)$ is closed for all $n \geq d$. For any $n \geq d$,

$$
M+R\left(T^{n}\right)=N\left(T^{d}\right)+N+R\left(T^{n}\right)=N\left(T^{d}\right)+R\left(T^{n}\right),
$$

which is a closed subspace by [4, Theorem 3.2]. Clearly $M \cap R\left(T^{n}\right)=N$ is also a closed subspace. Now both $M$ and $R\left(T^{n}\right)$ are paracomplete [6, Prop. 2.1.4]. Using the Neubauer Lemma [6, Prop. 2.1.2], we deduce that $R\left(T^{n}\right)$ is closed. Hence $T$ is quasi-Fredholm.

Conversely, assume $T$ is quasi-Fredholm with uniform descent $d$. Take $N=$ $R\left(T^{\infty}\right)$ and $M=N\left(T^{d}\right)+R\left(T^{\infty}\right)$. It is clear that $M$ and $N$ are $T$-invariant subspaces. It is also clear that $N \subseteq M$ and $T^{d} M \subseteq N$. Since $R\left(T^{n}\right)$ is closed for $n \geq d$ and $N=R\left(T^{\infty}\right), N$ is closed. Moreover, we have $T N=N$ [4, Theorem 3.4]. So $T_{N}$ is surjective and $T^{-n} N=N+N\left(T^{n}\right)$ for all $n$. By Lemma 6 , we have $M=N+N\left(T^{n}\right)=T^{-n} N$ for all $n \geq d$. It follows that $M$ is closed and $T^{-1} M=M$. Hence, $T_{X / M}$ is injective. Also,

$$
R(T)+M=R(T)+N+N\left(T^{d}\right)=R(T)+N\left(T^{d}\right),
$$

which is a closed subspace by the definition of topological uniform descent. We conclude that $R\left(T_{X / M}\right)$ is closed and hence $T_{X / M}$ is bounded below.

Theorem 8. If $\operatorname{des}(T)=d$ and $R\left(T^{d}\right)$ is closed, then the stability radius of $T$ is given by $\delta(T)=\lim _{n \rightarrow \infty} \gamma\left(T^{n}\right)^{1 / n}$.

Remark. We readily deduce from [4, Corollary 4.8 (c)] that $T-\lambda I$ is surjective for every $\lambda \in \Omega$, where $\Omega$ is the component of $\rho_{\gamma}(T)$ defined in Lemma 6 .

Proof. We assume that $T^{d} \neq 0$; otherwise both $\delta(T)$ and $\lim _{n \rightarrow \infty} \gamma\left(T^{n}\right)^{1 / n}$ are infinite. Let $M=R\left(T^{d}\right)=R\left(T^{\infty}\right)$. It is then easy to verify that $T_{M}$ is surjective and $T_{X / M}$ is nilpotent. By Lemma 3 , there is positive real number $\delta$ such that

$$
\delta=\lim _{n \rightarrow \infty} \gamma\left(T^{n}\right)^{1 / n}=\lim _{n \rightarrow \infty} \gamma\left(T_{M}^{n}\right)^{1 / n} .
$$

We know from [8] that $\delta$ is the surjectivity radius of $T_{M}$. We proceed to show that $\delta=\delta(T)$. If $0<|\lambda|<\delta$, then $(T-\lambda I)_{M}$ is surjective. Since $T_{X / M}$ is nilpotent, $(T-\lambda I)_{X / M}$ is invertible and hence bounded below. Using Lemma 5 , we deduce that $T-\lambda I$ is s-regular. Hence, $\delta \leq \delta(T)$.

Conversely, assume $0<|\lambda|<\delta(T)$. Since $T_{X / M}$ is nilpotent, $(T-\lambda I)_{X / M}$ is invertible and $(T-\lambda I)^{-1} M=M$. It follows that

$$
(T-\lambda I) M=(T-\lambda I)(T-\lambda I)^{-1} M=M \cap R(T-\lambda I) .
$$

It is routine to verify that $T$ is quasi-Fredholm. Thus, $M=R\left(T^{\infty}\right) \subseteq R(T-\lambda I)$ by Lemma 6 . So we have $(T-\lambda I) M=M$. This shows that the surjectivity radius of $T_{M}$ is no less than $\delta(T)$. Hence, $\delta(T) \leq \delta$.

A dual to the above theorem is the following. 
Theorem 9. If $\operatorname{asc}(T)=d$ and $R\left(T^{n}\right)$ is closed for all $n \geq d$, then the stability radius of $T$ is given by $\delta(T)=\lim _{n \rightarrow \infty} \gamma\left(T^{n}\right)^{1 / n}$.

Theorem 10. Let $T$ be a quasi-Fredholm operator; then the stability radius of $T$ is given by $\delta(T)=\lim _{n \rightarrow \infty} \gamma\left(T^{n}\right)^{1 / n}$.

Proof. Let $M=R\left(T^{\infty}\right)+N\left(T^{d}\right), N=R\left(T^{\infty}\right)$. It follows from Theorem 7 that the subspaces $M$ and $N$ satisfy the requirement for Lemma 4 . We now have:

(a) $T_{X / M}$ is bounded below and $\delta\left(T_{X / M}\right)=\lim _{n \rightarrow \infty} \gamma\left(T_{X / M}^{n}\right)^{1 / n}$.

(b) By Lemma 4 (iii), $T_{M}$ satisfies the requirement for Theorem 8 and

$$
\delta\left(T_{M}\right)=\lim _{n \rightarrow \infty} \gamma\left(T_{M}^{n}\right)^{1 / n}=\lim _{n \rightarrow \infty} \gamma\left(T_{N}^{n}\right)^{1 / n} .
$$

(c) By (a), (b) and Lemma 4 (v), the limit $\delta=\lim _{n \rightarrow \infty} \gamma\left(T^{n}\right)^{1 / n}$ exists and

$$
\delta=\min \left\{\delta\left(T_{X / M}\right), \delta\left(T_{M}\right)\right\} .
$$

If $0<|\lambda|<\delta$, then $|\lambda|$ is less than both $\delta\left(T_{X / M}\right)$ and $\delta\left(T_{M}\right)$. Thus $(T-\lambda I)_{X / M}$ is bounded below [8] and $(T-\lambda I)_{M}$ is surjective (see the remark for Theorem 8). Lemma 5 shows that $T-\lambda I$ is s-regular. Hence, $\delta \leq \delta(T)$.

Conversely, let $0<|\lambda|<\delta(T)$. By Theorem $6, M=R\left[(T-\lambda I)^{\infty}\right]$. Since $T-\lambda I$ is s-regular, it follows from Theorem 5 that $(T-\lambda I)_{X / M}$ is bounded below and $(T-\lambda I)_{M}$ is surjective. Therefore,

$$
|\lambda|<\min \left\{\delta\left(T_{X / M}\right), \delta\left(T_{M}\right)\right\}=\delta .
$$

This shows that $\delta(T) \leq \delta$.

So far, the results obtained are independent of the Apostol representation of operators given in [12]. For the rest of this paper, we assume the following results from [12]. Let

$$
\mathcal{N}=\bigvee_{\lambda \in \rho_{\gamma}(T)} N(T-\lambda I) \quad \text { and } \quad \mathcal{R}=\bigcap_{\lambda \in \rho_{\gamma}(T)} R(T-\lambda I)
$$

So $\mathcal{N}$ is the closed subspace generated by $N(T-\lambda I)$ and $\mathcal{R}$ is the intersection of $R(T-\lambda I)$ over all $\lambda \in \rho_{\gamma}(T)$. Then $\mathcal{N}, \mathcal{R}$ are hyper-invariant subspaces of $T$ with $\mathcal{N} \subseteq \mathcal{R}$. If $T_{\delta}, T_{0}, T_{\pi}$ are the maps induced by $T$ on the spaces $\mathcal{N}, \mathcal{R} / \mathcal{N}, X / \mathcal{R}$ respectively, then the following properties hold.

(i) $T_{\delta}$ has dense range, $T_{\pi}$ is injective.

(ii) $T$ is s-regular if and only if $T_{\delta}$ is surjective, $T_{0}$ is invertible and $T_{\pi}$ is bounded below.

These two facts are required for the proofs of the following theorems. In particular, we need to know that $\sigma\left(T_{0}\right) \subseteq \sigma_{\gamma}(T)$, which is a consequence of (ii). It is also known that $T$ is quasi-Fredholm if and only if $T_{\delta}$ is surjective, $T_{0}$ is chain finite and $T_{\pi}$ is bounded below. However, we will not use this fact.

Lemma 11. Let $N \subseteq M$ be closed T-invariant subspaces of $X$. Suppose that $T_{X / M}$ is injective, $T^{\bar{d}} M \subseteq N$ for some $d$ and $T_{N}$ has dense range. If the limit $\lim \sup _{n \rightarrow \infty} \gamma\left(T^{n}\right)^{1 / n}$ is non-zero, then $T$ is quasi-Fredholm.

Proof. In the light of Theorem 7 , it suffices to prove that $T_{X / M}$ is bounded below and $T_{N}$ is surjective. It is clear from Lemma 1 that both $\lim _{\sup } \rightarrow \infty \gamma\left(T_{X / N}^{n}\right)^{1 / n}$ and $\lim _{\sup } \operatorname{si\infty }_{n \rightarrow \infty} \gamma\left(T_{M}^{n}\right)^{1 / n}$ are positive. 
Since $T^{d} M \subseteq N$ and $T_{X / M}$ is injective, we have the inclusions

$$
M \subseteq T^{-n} N \subseteq T^{-n} M \subseteq M
$$

for each $n \geq d$. Hence $M=T^{-n} N$ and $N\left(T_{X / N}^{n}\right)=M / N$ for $n \geq d$. Applying Lemma 2 to the operator $T_{X / N}$, we deduce that $\left(T_{X / N}\right)^{\wedge}$ and hence $T_{X / M}$ is bounded below.

Since $T_{N}$ has dense range, so has $T_{N}^{n}$ for each $n \geq d$. Therefore

$$
N \subseteq \overline{T^{n} N} \subseteq \overline{T^{n} M} \subseteq \bar{N}=N .
$$

Hence, $\overline{R\left(T_{M}^{n}\right)}=\overline{R\left(T_{M}^{d}\right)}=N$ for $n \geq d$. Applying Lemma 3 to the operator $T_{M}$, we deduce that $\left(T_{M}\right)^{\sim}=T_{N}$ is surjective.

The proofs of the following two lemmas are elementary, so we omit them.

Lemma 12. Let $N \subseteq M$ be closed $T$-invariant subspaces of $X$. If both $T_{X / M}$ and $T_{M / N}$ are injective, then $T_{X / N}$ is also injective.

Lemma 13. Let $M_{1}, M_{2}$ be $T$-invariant closed subspaces of $X$ such that $M_{1}+M_{2}$ is closed. Let

$$
X_{1}=\frac{M_{1}}{M_{1} \cap M_{2}}, X_{2}=\frac{M_{2}}{M_{1} \cap M_{2}}, \quad Y_{1}=\frac{M_{1}+M_{2}}{M_{1}}, Y_{2}=\frac{M_{1}+M_{2}}{M_{2}} .
$$

Then the diagrams

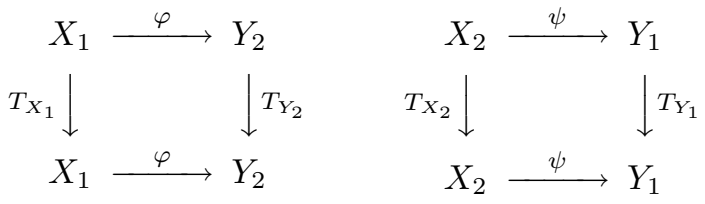

are commutative, and $\varphi$ and $\psi$ are linear homeomorphisms induced by the identity on $X$.

We now give a generalization of [1, Prop. 3.3].

Theorem 14. Let $\sigma$ be a closed and open subset of $\sigma_{\gamma}(T)$. If $0 \in \sigma$ and

$$
\limsup _{n \rightarrow \infty} \gamma\left(T^{n}\right)^{1 / n}=r>\sup _{\lambda \in \sigma}|\lambda|
$$

then $T$ is quasi-Fredholm.

Proof. Since $T_{\delta}$ has dense range and $T_{\pi}$ is injective, we can apply both part (i) and (ii) of Lemma 1 to show that $\gamma\left(T_{0}^{n}\right) \geq \gamma\left(T^{n}\right)$ for all $n$. Let $\sigma_{1}=\sigma \cap \sigma\left(T_{0}\right)$, $\sigma_{2}=\left[\sigma_{\gamma}(T) \backslash \sigma\right] \cap \sigma\left(T_{0}\right)$; then both $\sigma_{1}$ and $\sigma_{2}$ are closed. Since $\sigma\left(T_{0}\right) \subseteq \sigma_{\gamma}(T)$, we have $\sigma_{1} \cup \sigma_{2}=\sigma\left(T_{0}\right)$. Hence $\sigma_{1}$ and $\sigma_{2}$ are spectral sets of $T_{0}$. The spectral sets induce a decomposition

$$
\mathcal{R} / \mathcal{N}=X_{1} \oplus X_{2}, \quad T_{0}=T_{1} \oplus T_{2} \quad \text { with } \sigma\left(T_{1}\right)=\sigma_{1}, \sigma\left(T_{2}\right)=\sigma_{2} .
$$

Since $0 \notin \sigma_{2}, T_{2}$ is invertible. We claim that $T_{1}$ is nilpotent. Let us assume that $\sigma_{1} \neq \emptyset$; otherwise, $X_{1}=0$ and $T_{1}$ is trivially nilpotent. For each $x_{1} \in X_{1}, x_{2} \in X_{2}$,

$$
T_{0}\left(x_{1}+x_{2}\right) \in X_{1} \Rightarrow T_{2} x_{2} \in X_{1} \Rightarrow T_{2} x_{2} \in X_{1} \cap X_{2}=\{0\} \Rightarrow x_{2}=0 .
$$

Therefore, $T_{0}^{-1} X_{1} \subseteq X_{1}$. Thus $T_{0}$ and $X_{1}$ satisfy part (i) of Lemma 1 . Hence, $\gamma\left(T_{1}^{n}\right) \geq \gamma\left(T_{0}^{n}\right) \geq \gamma\left(T^{n}\right)$ for all $n$. Taking limits,

$$
r=\limsup _{n \rightarrow \infty} \gamma\left(T^{n}\right)^{1 / n} \leq \limsup _{n \rightarrow \infty} \gamma\left(T_{1}^{n}\right)^{1 / n} .
$$


It is well-known that if $S \neq 0$, then $\gamma(S) \leq\|S\|$. So if $T_{1}$ is not nilpotent, then

$$
r \leq \limsup _{n \rightarrow \infty} \gamma\left(T_{1}^{n}\right)^{1 / n} \leq \lim _{n \rightarrow \infty}\left\|T_{1}^{n}\right\|^{1 / n}=\sup \left\{|\lambda|: \lambda \in \sigma_{1}\right\} .
$$

Note that the equality on the right comes from the spectral radius formula and the fact that $\sigma\left(T_{1}\right)=\sigma_{1}$. This contradicts (6). Hence, $T_{1}$ is nilpotent. This proves our claim.

Suppose $X_{1}=M_{1} / \mathcal{N}$ and $X_{2}=M_{2} / \mathcal{N}$. Since $\mathcal{R} / \mathcal{N}=X_{1} \oplus X_{2}$, it is not difficult to see that

$$
M_{1} \cap M_{2}=\mathcal{N} \quad \text { and } \quad M_{1}+M_{2}=\mathcal{R} .
$$

In the context of Lemma 13, we have $T_{Y_{1}}=T_{\mathcal{R} / M_{1}}, T_{X_{2}}=T_{2}$ and $T_{\mathcal{R} / M_{1}}=$ $\psi T_{2} \psi^{-1}$. Since $T_{2}$ is invertible, so is $T_{\mathcal{R} / M_{1}}$. By definition, $T_{X / \mathcal{R}}$ equals $T_{\pi}$, which is always injective. Thus, both $T_{X / \mathcal{R}}$ and $T_{\mathcal{R} / M_{1}}$ are injective, and so is $T_{X / M_{1}}$ by Lemma 12. Moreover, $T_{M_{1} / \mathcal{N}}=T_{1}$ is nilpotent and $T_{\mathcal{N}}=T_{\delta}$ have dense range. Using Lemma 11, we deduce that $T$ is quasi-Fredholm.

Corollary 15. Let 0 be an isolated point of $\sigma_{\gamma}(T)$; then the limit $\lim _{n \rightarrow \infty} \gamma\left(T^{n}\right)^{1 / n}$ always exists. This limit is non-zero if and only if $T$ is quasi-Fredholm.

Proof. Let $\eta=\lim \sup _{n \rightarrow \infty} \gamma\left(T^{n}\right)^{1 / n}$. We have two cases.

(a) If $\eta=0$, then $\lim _{n \rightarrow \infty} \gamma\left(T^{n}\right)^{1 / n}=0$.

(b) If $\eta>0$, we can put $\sigma=\{0\}$, and $T$ is quasi-Fredholm by Theorem 14 .

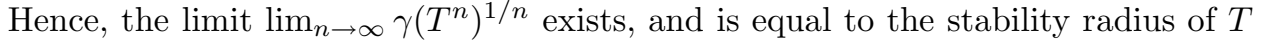
by Theorem 10 .

Therefore, the $\operatorname{limit}_{\lim } \rightarrow \infty \gamma\left(T^{n}\right)^{1 / n}$ always exists and equals $\eta$. If $\eta \neq 0$, then case (b) must be true and $T$ is quasi-Fredholm. Conversely, if $T$ is quasi-Fredholm, then $\eta$ is the stability radius of $T$. From Lemma 6 , we know that the stability radius of a quasi-Fredholm operator is always non-zero. This completes our proof.

The significance of the corollary is that the class of quasi-Fredholm operators is the most general class of operators for which the $\operatorname{limit}_{n \rightarrow \infty} \gamma\left(T^{n}\right)^{1 / n}$ is equal to the stability radius $\delta(T)$ with respect to the Apostol spectrum.

\section{ACKNOWLEDGEMENT}

The author wishes to thank the referee for suggesting improved organization of the paper as well as a revision and clarification of some proofs.

\section{REFERENCES}

1. C. Apostol, The reduced minimum modulus, Michigan Math. J. 32 (1985), 279-294. MR 87a: 47003

2. H. Bart and C. Lay, The stability radius of a bundle of closed linear operators, Studia Math. 66 (1980), 307-320. MR 82c:47014

3. K.-H. Förster and M. A. Kaashoek, The asymptotic behaviour of the reduced minimum modulus of a Fredholm operator, Proc. Amer. Math. Soc. 49 (1975), no. 1, 123-131. MR 51:8867

4. S. Grabiner, Uniform ascent and descent of bounded operators, J. Math. Soc. Japan 34 (1982), no. 2, 317-337. MR 84a:47003

5. V. Kordula and V. Müller, The distance from the Apostol spectrum, Proc. Amer. Math. Soc. 124 (1996), 3055-3061. MR 96m:47007

6. J. Ph. Labrousse, Les opérateurs quasi Fredholm: Une généralisation des opérateurs semi Fredholm, Rend. Circ. Mat. Palermo (2) 29 (1980), 161-258. MR 83c:47022

7. J. Ph. Labrousse and M. Mbekhta, Résolvant généralisé et séparation des points singuliers quasi-Fredholm, Trans. Amer. Math. Soc. 333 (1992), no. 1, 299-313. MR 92k:47007 
8. E. Makai and J. Zemánek, The surjectivity radius, packing numbers and boundedness below of linear operators, Integral Equations Operator Theory 6 (1983), 372-384. MR 84m:47005

9. M. Mbekhta and V. Müller, On the axiomatic theory of spectrum II, Studia Math. 119 (1996), 129-147. MR 97c:47005

10. M. Mbekhta and A. Ouahab, Contribution à la théorie spectrale généralisée dans les espaces de Banach, C. R. Acad. Sci. Paris Sér. I Math. 313 (1991), 833-836.

11. V. Müller, On the regular spectrum, J. Operator Theory 31 (1994), 363-380.

12. P. W. Poon, The Apostol representation of a linear operator, Preprint, Department of Mathematics, University of Melbourne.

13. Ch. Schmoeger, The stability radius of an operator of Saphar type, Studia Math. 113 (1995), no. 2, 169-175. MR 96a:47019

14. J. Zemánek, The stability radius of a semi-Fredholm operator, Integral Equations Operator Theory 8 (1985), 137-144. MR 86c:47014

Department of Mathematics, University of Melbourne, Victoria, 3052, Australia

E-mail address: pakpoon@maths.mu.oz.au 